\title{
Convergencia de las Comunicaciones Móviles hacia Sistemas LTE y LTE-A de Cuarta Generación
}

\section{Mobile Communications Convergence to LTE and LTE-A Systems of Fourth Generation}

\author{
Jhovany Santacruz ${ }^{1}$ y Rafael García ${ }^{2}$ \\ ${ }^{1}$ Carrera de Ingeniería de Sistemas, Universidad Católica de Cuenca \\ Cañar, 030350, Ecuador \\ ${ }^{2}$ Universidad Católica de Cuenca, Cuenca, 010105, Ecuador \\ *jsantacruze@ucacue.edu.ec
}

\begin{abstract}
Resumen
Este artículo plantea un análisis histórico de la vertiginosa evolución que han tenido y mantienen los sistemas de comunicaciones móviles y sus tecnologías subyacentes. En principio se realiza una descripción breve, pero didáctica de las diferentes tecnologías asociadas a cada una de las generaciones que han aparecido en términos de su evolución cronológica, dejando en claro los beneficios que suponen, así como sus principales limitantes que han dado paso a otros tipos de implementaciones encaminadas a mejorar las tasas de transferencia de datos, manejo de medios y la movilidad del usuario. Finalmente, se brinda una descripción, no muy exhaustiva, pero lo suficientemente detallada para su entendimiento, de las tecnologías LTE y su evolución hacia LTE-Advanced, como referentes de la cuarta generación de móviles (4G), actualmente la más difundida, y sobre cuya base continua un marcado proceso de desarrollo e innovación.
\end{abstract}

Palabras clave: LTE, LTE-A, comunicaciones móviles, redes celulares, generaciones móviles.

\begin{abstract}
This article presents a historical analysis of the vertiginous evolution that have had, and still have, the mobile communication systems and their subjacent technologies. To begin with, a brief but didactic description of the technologies associated to each of the generations that have appeared in terms of their chronological evolution is made, making clear the implied benefits, as well as their main limitations which had led to other types of implementations aimed at improving data transfer rates, the media management, and user's mobility. Finally, a not very exhaustive, but sufficiently detailed description for the understanding of the LTE technologies and their evolution towards "LTE-Advanced", as references of the fourth generation of mobiles $(4 G)$ is given. Currently, the most widespread, and on the basis of a marked process of development and innovation.
\end{abstract}

Key words: LTE, LTE-A, mobile communications, celular networks, mobile generations.

\section{INTRODUCCIÓN}

M Ás de dos décadas han transcurrido desde los inicios de lo que hoy en día conocemos como redes celulares y teléfonos móviles; hecho que tuvo su primer antecedente a raíz de la construcción del primer dispositivo de comunicación de esta naturaleza por la compañía Motorola, por el año de 1983.

De ahí en adelante se ha tenido un ininterrumpido crecimiento y avance de la industria de las comunicaciones móviles que ha obtenido cada vez más usuarios en todo el mundo, llegando a ser hoy en día un elemento indispensable para nuestras vidas, satisfaciendo la enorme necesidad de comunicación y movilidad, que por mucho fue un sueño para el ser humano.

A lo largo del camino han surgido tendencias tecnológi- cas, en términos de infraestructura y servicios de comunicación, que para mejorar su entendimiento e identificación, han sido nombradas como una serie de generaciones.

Cada generación supone un avance tecnológico que ha permitido mejorar e integrar nuevos servicios con respecto a las generaciones anteriores.

Hasta la fecha han sido lanzadas cuatro generaciones móviles representadas cada una por una serie de características técnicas e integración de nuevos servicios, las mismas que sus últimas versiones denotan una convergencia evidente hacia estrategias de comunicación basadas en el protocolo IP, con altas tasas de transferencia, tanto en modo estacionario, como con usuarios móviles, mejorando la seguridad, y todo al mínimo costo posible. 


\section{EVOLUCIÓN DE LAS COMUNICACIONES MÓVILES}

La primera generación $(1 \mathrm{G})$ está fundamentalmente representada por la tecnología AMPS (Advanced Mobile Phone System), se basa en un sistema analógico con baja capacidad de roaming, permitía solo llamadas de voz de baja calidad, apareció por primera vez en los Estados Unidos en 1983 [1]. Su núcleo está basado en una red de conmutación de circuitos, y aunque su uso esté descontinuado, ha sido un sistema de importancia histórica para el desarrollo de las comunicaciones móviles por el éxito obtenido y por las innovadoras ideas que aportaba.

La segunda generación (2G), que llegó a partir de 1990, implica un cambio significativo con respecto a la anterior, y es la migración del sistema analógico a un sistema digital, lo cual supone mejor calidad de voz, mayor seguridad y reducción de costos por simplificarse la construcción de los terminales. En $2 \mathrm{G}$ se sigue utilizando una red de conmutación de circuitos, pero con mejoras en la calidad de la voz, capacidades mejoradas de roaming y se integran los primeros servicios de valor agregado como los mensajes cortos SMS. El referente tecnológico de esta generación es GSM (Global System for Mobile Communications), que utiliza TDMA (Time Division Multiple Access) y FDMA (Frequency Division Multiple Access) como técnicas de control de acceso al medio, para solventar el problema de un reducido espectro radioeléctrico disponible, permitiendo así un incremento en el número de usuarios simultáneos. A la segunda generación también se le atribuye otros protocolos como IS- 136 o TDMA, IS-95 o cdmaONE, DAMPS (la versión digital de AMPS 1G) y PHS [2]; todos éstos de diferentes propietarios e incompatibles entre sí, implementados en distintas regiones del mundo, limitaban el uso de los móviles solo a su área de cobertura. Fue GSM entonces la tecnología que tuvo un éxito abrumador frente a sus competidores, y es así que terminó imponiéndose, ofreciendo una cobertura potencialmente mundial, con millones de abonados suscritos a las operadoras que la soportan.

La tercera generación no llegó inmediatamente, ya que muchos suelen asignar el calificativo de $2.5 \mathrm{G}$ y $2.75 \mathrm{G}$ a tecnologías representadas por GPRS y EDGE respectivamente. Lo que muestran en realidad son una transición entre la segunda y tercera generación de móviles que implican algunas mejoras implementadas sobre los servicios 2G. Con GPRS se alcanzaban velocidades entre 54 y 114 kpbs, permitiendo el uso de servicios como WAP (Wireless Application Protocol), sistema de mensajería multimedia (MMS), email y acceso a la web, una verdadera revolución para esa época. Por otro lado aparace EDGE (Enhanced Data Rates for GSM Evolution) como una mejora a GPRS (EGPRS), permitiendo que aplicaciones con altos requerimientos de ancho de banda, como video y multimedia, puedan entrar en la escena de los móviles. EDGE puede alcanzar una velocidad de transmisión de $384 \mathrm{kpps}$ en modo de conmutación de paquetes, triplicando a GPRS, y es por esto que se le puede considerar el punto más cercano hacia la tercera generación [3] [4].
Poco después vio la luz la tercera generación (3G) caracterizada del mismo modo que sus predecesoras por una serie de tecnologías y estándares de distintos competidores en la lucha por lograr predominio en un mercado tan competitivo como el de las telecomunicaciones. Sin embargo, a diferencia de $2 \mathrm{G}$, la tercera generación fue estandarizada por el proceso IMT-2000, el cual regularizó no solo una tecnología, sino un conjunto de especificaciones y requerimientos para $3 \mathrm{G}$, entre los cuales tenemos las tasas máximas de transferencia ( $2 \mathrm{Mbps}$ en ambientes estacionarios y $384 \mathrm{Kbps}$ en movimiento). 3G representa un cambio significativo que aumenta la capacidad para la transmisión de datos en movilidad y la llegada de la banda ancha a las comunicaciones móviles. Uno de los estándares más difundidos de esta generación que cumple con las especificaciones IMT-2000, es UMTS (Universal Mobile Telecommunication System), que usa W-CDMA (CDMA de banda ancha) como interfaz de aire, lo cual facilita enormemente alcanzar mayores velocidades con respecto a GSM de segunda generación que utilizaba TDMA como técnica de control de acceso al medio. La gran mayoría de los móviles estaban usando redes UMTS. Existen otros estándares de tercera generación como CDMA 2000, pero que no fue tan aceptado como UMTS a nivel global. Del mismo modo, que en la segunda generación, se han ido dando avances y mejoras en $3 \mathrm{G}$ a lo largo de su convergencia hacia 4G, y es así como de forma comercial se han ido creando nombres intermedios como $3.5 \mathrm{G}$ para referirse a tecnologías como HSDPA (velocidades de bajada de hasta 14.4 Mbps), HSUPA (con velocidades de subida de hasta $5.8 \mathrm{Mbps}$ ) o $3.75 \mathrm{G}$ para hacer referencia a HSPA+ que no es otra más que una evolución de HSPA que mejora las tasas de subida y de bajada; comercialmente se lo puede denominar como 4G, pero tecnológicamente la cuarta generación comienza, o al menos se acerca, con LTE [2]. Como se puede apreciar son denominaciones más comerciales que estándares formalmente aceptados, que incluso pueden diferir entre diversos autores y operadoras.

\section{LTE Y LTE-A, LA EVOLUCIÓN HACIA 4G}

Luego de la descripción histórica y evolutiva de las generaciones móviles y sus tecnologías relacionadas, llegamos al punto de inicio de la cuarta generación.

En la Fig. 1 se observa que no existe un solo grupo de interés encargado de la estandarización, pero es quizá 3GPP el principal organismo destinado para el efecto, y al seguir su evolución tecnológica se aprecia que LTE y su convergencia a LTE- Advanced, representan los estándares de referencia para la cuarta generación de comunicaciones móviles.

\section{A. Procesos de estandarización}

Las tecnologías de la información y comunicación en general y las comunicaciones móviles en particular, tienen un alto grado de incidencia en el crecimiento económico y la productividad, el terminal móvil es parte de nuestra 


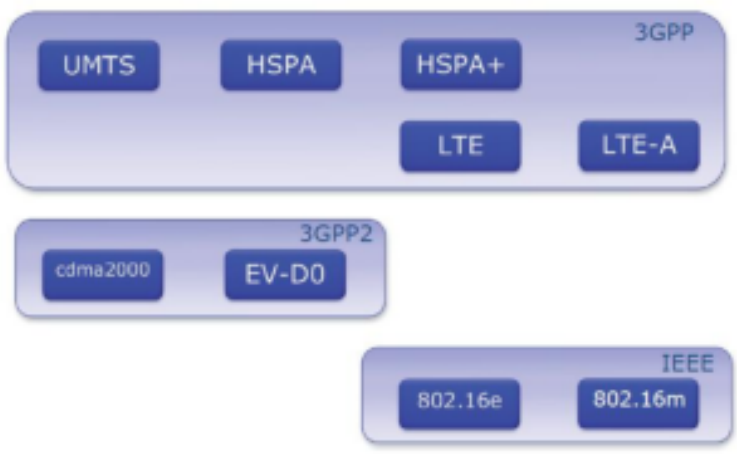

FIG. 1. Panorámica de sistemas móviles 3G/4G.

diaria existencia; es por ello que los distintos organismos y foros de estandarización adquieren un rol fundamental en el contexto de su desarrollo y difusión.

Los procesos de estandarización requieren de mucho tiempo y esfuerzo, parten de las especificaciones iniciales que debe cumplir el sistema, luego se define la arquitectura, se hacen las pruebas correspondientes hasta alcanzar un alto grado de madurez y estabilidad, todo esto en el marco de un proceso iterativo en el que cada vez añaden o eliminan nuevas especificaciones técnicas. Así es como surgió el nuevo estándar de comunicaciones móviles LTE (ya en su proceso maduro) y su correspondiente evolución hacia LTE-A.

\section{B. LTE (Long Term Evolution)}

Las especificaciones para LTE surgen del 3GPP, que nació en 1998. Los documentos del 3GPP se estructuran en Releases, cada una caracterizada por la incorporación de mejoras con respecto a la versión anterior. (Ver tabla I).

Así, la denominada R99 (diciembre de 1999) supuso el primer conjunto de especificaciones para UMTS, seguido tras la llamada R4, se completó en marzo de 2002 la R5 que incluye por ejemplo HSDPA, tres años más tarde se incluye HSUPA en R6, en la R7 (septiembre de 2007) se incluye HSPA+, mientras que LTE/SAE se asocian a R8 y posteriores. Para una referencia más completa de las diferentes versiones, referirse a la tabla 1 , donde se indica el proceso evolutivo de la tecnología móvil en términos de estandarización [5].

\section{Arquitecturas de implementaciones previas}

Previo a realizar un análisis de la estructura técnica de LTE, es conveniente que se analice la arquitectura genérica adoptada por los sistemas $2 \mathrm{G} / 3 \mathrm{G}$ definidos por $3 \mathrm{GPP}$, lo que permite establecer de forma clara las mejoras que supone LTE con respecto a sistemas GSM y UMTS.

En la Fig. 2 se podrá analizar la arquitectura genérica de un sistema celular.

Como se puede apreciar en el esquema se cuenta con tres elementos básicos:
TABLA I

\begin{tabular}{|c|c|}
\hline \multicolumn{2}{|c|}{ VERSIONES DEL PROYECTO 3GPP } \\
\hline Release & Información \\
\hline $\mathrm{Ph} \mathrm{I}$ & Características del Sistema GSM \\
\hline $\mathrm{Ph} 2$ & Características del sistema GSM, Códec EFR \\
\hline R96 & $\begin{array}{l}\text { Características del sistema GSM, } 14.4 \mathrm{kbit} / \mathrm{s} \text { de ancho } \\
\text { de banda para el usuario }\end{array}$ \\
\hline R97 & Características del sistema GSM, Aparición del GPRS \\
\hline R99 & $\begin{array}{l}\text { Aparición de la primera red de tercera generación } \\
\text { UMTS, incorporando una interfaz de aire CDMA }\end{array}$ \\
\hline Rel-4 & $\begin{array}{l}\text { Originalmente llamada Versión } 2000-\text { añadía } \\
\text { características como una Red de núcleo all-IP }\end{array}$ \\
\hline Rel-5 & Introdujo el IMS y HSDPA \\
\hline Rel-6 & $\begin{array}{l}\text { Operación integrada con Redes LAN Wireless LAN } \\
\text { y añadia HSUPA, MBMS, mejoras al IMS como } \\
\text { Push to Talk over Cellular (PoC) y GAN }\end{array}$ \\
\hline Rel-7 & $\begin{array}{l}\text { Esta versión se centró en rebajar la latencia, } \\
\text { mejorar la Calidad de Servicio y el uso de } \\
\text { aplicaciones en tiempo real como por ejemplo la Voz } \\
\text { sobre IP VoIP. Esta versión también se centró en el } \\
\text { desarrollo de la red HSPAt, una mejora en el ancho } \\
\text { de banda de los sistemas GSM EDGE, el protocolo } \\
\text { de alta velocidad SIM y el protocolo de } \\
\text { comunicación sin contacto (Near Field } \\
\text { Communication que permitió a los operadores } \\
\text { ofrecer servicios como por ejemplo el pago a través } \\
\text { del móvil) }\end{array}$ \\
\hline Rel-8 & $\begin{array}{l}\text { Aparece la primera versión de red LTE así como } \\
\text { la red All-IP (SAE). Aparecen también las nuevas } \\
\text { interfaces de radio OFDMA, FDE y MIMO que no } \\
\text { serán compatibles con las redes basadas en CDMA, } \\
\text { encontramos una primera especificación del Dual- } \\
\text { Cell HSDPA }\end{array}$ \\
\hline Rel-9 & $\begin{array}{l}\text { Mejoras de la red SAE, aparición del WiMAX y } \\
\text { compatibilidad entre redes LTE y UMTS. Se sigue con } \\
\text { el desarrollo del Dual-Cell HSDPA con MIMO, y } \\
\text { aparece el Dual-Cell HSUPA }\end{array}$ \\
\hline Rel-10 & $\begin{array}{l}\text { Aparece el LTE avanzado LTE-Advanced } \\
\text { cumpliendo los requisitos del IMT Advanced } 4 \mathrm{G} \text {. } \\
\text { Esta nueva especificación será compatible con el } \\
\text { LTE desarrollado en la versión } 8 \text {. Aparece la } \\
\text { tecnología Multe-Cell HSDPA ( } 4 \text { portadoras) }\end{array}$ \\
\hline Rel-11 & $\begin{array}{l}\text { Interconexión IP de servicios avanzado, interconexión } \\
\text { en la capa de servicio entre operadores nacionales } \\
\text { y proveedores de aplicaciones }\end{array}$ \\
\hline Rel-12 & $\begin{array}{l}\text { Fue completamente terminado en marzo de } 2015 \text {, su } \\
\text { prioridad fue el uso de la tecnología LTE para } \\
\text { servicios de seguridad. Otra característica importante } \\
\text { de este release incluye: celdas pequeñas y } \\
\text { densificación de red. }\end{array}$ \\
\hline Rel-13 & $\begin{array}{l}\text { (LTE-U) LTE sin licencia, mejoras LTE para } \\
\text { comunicaciones de tipo máquina, posicionamiento en } \\
\text { interiores. }\end{array}$ \\
\hline Rel-14/15 & Evolución a $5 \mathrm{G}$ \\
\hline
\end{tabular}

- El equipo de usuario que permite acceder a los servicios de red

- La red de acceso (celdas), parte del sistema responsable de sustentar la transmisión de radio con los equipos del usuario, permitiendo que éstos lleguen a la red troncal.

- La Red Troncal, es la parte del sistema que gestiona el acceso a la red celular, la movilidad de los usuarios y los mecanismos de interconexión con otras redes, 


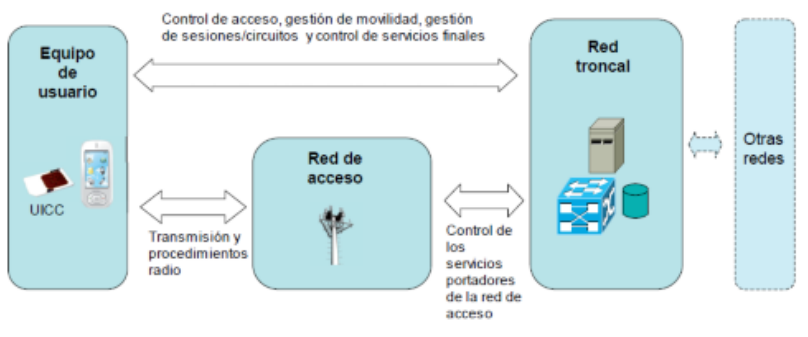

FIG. 2. Arquitectura genérica de un sistema celular.

tales como la PSTN u otras operadoras móviles. Está conformada por equipos que soportan funciones de conmutación de circuitos, encaminamientos de paquetes (routing), bases de datos, etc.

Esta arquitectura ha sido adoptada por las diferentes familias de sistemas celulares $2 \mathrm{G}$ y $3 \mathrm{G}$, y también se mantiene en LTE.

En la Fig. 3, se describe con más de detalle la arquitectura general de los sistemas propuestos por el foro 3GPP.

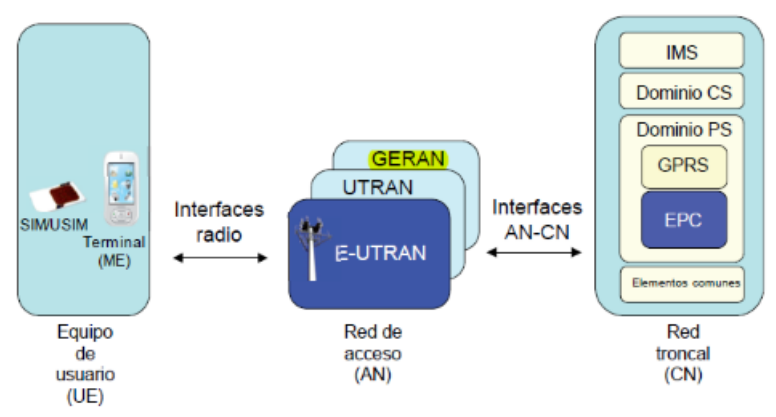

FIG. 3. Arquitectura general de los sistemas 3GPP.

Nótese como se mantiene el diseño arquitectónico genérico descrito, pero con ciertas separaciones lógicas en la red de acceso (Access Network, AN) y en la red troncal (Core Network, CN). Bajo esta arquitectura el equipo móvil se compone de dos partes: el propio dispositivo y una tarjeta UICC, denominada SIM en el caso de GSM o USIM para el caso de UMTS y LTE, y es la encargada de almacenar la información referente a la suscripción del usuario a los diferentes servicios proporcionados por la red.

En lo referente a la red de acceso (AN) 3GPP ha especificado tres tipos diferentes: GERAN (GSM/EDGE Radio Access Network), UTRAN (UMTS Terrestrial Radio Access Network) y E-UTRAN (Evolved-UTRAN). Las redes de acceso GERAN y UTRAN forman parte del sistema 3G/UMTS, mientras que E-UTRAN es la nueva red de acceso al sistema LTE. GERAN se le denomina eventualmente solo como GSM y utiliza un mecanismo de acceso al medio basado en TDMA, UTRAN utiliza WCDMA, y EUTRAN apuesta por la tecnología OFDMA, la cual provee de una mayor eficiencia en el uso del espectro [6].
La red troncal o Core Network, por su parte, se subdivide en forma lógica en un dominio de circuitos (CS Domain), un dominio de paquetes (PS Domain) y el subsitema IP Multimedia (IP Multimedia Subsystem, IMS).

El dominio CS contempla todos los elementos del Core capaces de proveer servicios basados en conmutación de circuitos a través de canales dedicados, tales como servicios de voz y videoconferencia en UMTS.El dominio de circuitos puede ser accedido a través de GERAN y UTRAN, en contraste con el diseño E-UTRAN, que ya no contempla el acceso a CS, debido a que todos los servicios son provistos mediante el dominio PS.

El dominio PS, contiene todos los elementos del core encargados de proveer servicios basados en conmutación de paquetes de datos, proporcionando conectividad a cualquier tipo de red que soporte este mecanismo, tales como redes IP o X.25. Dentro de este dominio se pueden distinguir dos tipos de implementaciones: GPRS y EPC. GPRS, desarrollada inicialmente en el contexto de las redes GSM y que también forma parte de los sistemas UMTS. Los servicios provistos por GPRS son accesibles mediante GERAN y UTRAN. Por otro lado tenemos la segunda implementación denominada EPC, que ha sido desarrollada en el contexto del sistema LTE. EPC representa una evolución de GPRS con optimizaciones para proporcionar servicios de conectividad IP a los usuarios a través de E-UTRAN, además de proporcionar interoperabilidad, soportando acceso a sus servicios mediante las otras redes de la familia $3 \mathrm{GPP}$, como GERAN y UTRAN, y más aún a través de redes no $3 \mathrm{GPP}$ como CDMA 2000 y Mobile Wimax.

En el core además existen una serie de elementos que implementan funciones asociadas a diferentes dominios, como la base de datos HSS (Home Suscriber Server) que contiene la información de los usuarios del sistema.

El subsitema IMS es otro componente de la red troncal, y comprende los elementos relacionados con la provisión de servicios IP multimedia basados en el protocolo SIP (Session Initiation Protocol). IMS utiliza como mecanismo de transporte los servicios de transferencia provistos por el dominio de conmutación de paquetes (PS), constituyéndose en una función de control que está independiente de las funciones de transporte de información, que están asignadas al dominio PS, cuya característica ha sido adoptada plenamente por LTE en concordancia con trabajos realizados por otros organismos de estandarización, orientado a las arquitecturas de los futuros sistemas de telecomunicaciones que estarán basadas netamente en conmutación de paquetes, a las que se ha denominado Next Generation Network (NGN) o redes de siguiente generación.

\section{Arquitectura LTE}

Basándose en la arquitectura de los sistemas 3GPP, en la Fig. 4, se muestra de manera simplificada la arquitectura de los sistemas LTE, denominada como Evolved Packet System (EPS). 


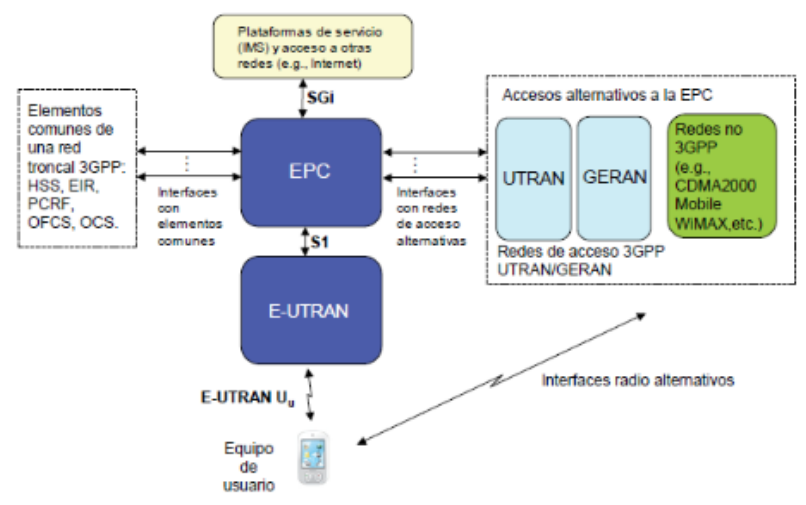

FIG. 4. Arquitectura de los sistemas LTE.

Los principales elementos o componentes de LTE son, por una parte, la nueva red de acceso E-UTRAN y el nuevo dominio de paquetes de la red troncal EPC, y por otra, se ve una evolución del subsistema IMS, diseñado en principio para sistemas UMTS. Estos elementos han sido diseñados de modo que puedan soportar todo tipo de servicios basados en conmutación de paquetes, por lo que no hace falta de ningún otro elemento que sea capaz de soportar servicios basados en conmutación de circuitos, ya que todos, incluidos aquellos servicios de tiempo real se proveen en base a la conmutación de paquetes, de modo que EPC puede ser visto como una evolución de GPRS.

La red de acceso E-UTRAN y la red troncal EPC proveen en conjunto el servicio de encaminado de paquetes entre el móvil o terminal del usuario y otras redes externas como Internet. De este modo se pueden diferenciar dos componentes del sistema LTE: EPS (EPS Bearer Service) que provee el servicio de transferencia de paquetes entre el equipo móvil del usuario y las redes externas y EUTRAN Radio Access Bearer (E-RAB) que hace referencia al servicio de transferencia de paquetes provisto por la propia red de acceso E-UTRAN.

En la figura 4 se pueden apreciar los diferentes tipos de interfaces que comunican los elementos del sistema LTE. La interfaz que une E-UTRAN y ECP se denomina S1 $\mathrm{y}$ proporciona los mecanismos para proveer acceso a los móviles a través de la red de acceso E-UTRAN. La interfaz de radio entre los dispositivos móviles y la E-UTRAN, se denomina E- UTRAN UU. Otra característica fundamental de LTE es que permite el acceso a sus servicios a través de GERAN y UTRAN (redes de acceso 3GPP), así como también mediante otras redes de acceso que no pertenecen a 3GPP, tales como CDMA 2000, Mobile Wimax y redes 802.11, proporcionando características de interoperabilidad al sistema, cuyas conexiones están soportadas por las interfaces de la EPC.

El núcleo del sistema LTE está basado en tecnologías IP, de modo que la infraestructura de una red LTE, aparte de los componentes propios que implementan las funcionalidades de 3GPP, integra otros componentes de las redes IP, tales como routers, servidores DHCP, para la configuración automática de los equipos en la red y Servidores DNS para asociar los nombres de los equipos con sus respectivas direcciones IP.

\section{E. Tecnologías de nivel físico en LTE}

En esta sección se presentan las tecnologías de nivel físico que dan soporte al sistema LTE y que representan uno de los cambios más significativos que han permitido alcanzar mayores niveles de eficiencia en cuanto a la capacidad general del sistema, así como a la gestión de sus recursos.

En primer lugar se deberá mencionar los fundamentos de las técnicas de acceso múltiple OFDM (Orthogonal Frequency Division Multiplex) y SC-FDMA, que se emplean para el enlace descendente y ascendente respectivamente.

La técnica de transmisión ODFM constituye un mecanismo de transmisión multiportadora que consiste en multiplexar un conjunto de símbolos sobre un conjunto de subportadoras. Debido a que cada subportadora es ortogonal se puede transmitir en simultáneo todos los símbolos con la capacidad que el receptor pueda diferenciar entre frecuencias que no le corresponden. Si bien es cierto OFDM es una técnica conocida desde hace muchos años, su uso en las comunicaciones móviles en más reciente, debido a que implica un cierto grado de complejidad para la implementación de los transmisores y receptores, ya ha sido utilizado en implementaciones de redes de área local IEEE 802.11 y hoy constituye la base de la técnica de acceso múltiple OFDMA que es utilizada por los sistemas LTE.

Esta tecnología divide el ancho de banda en múltiples bandas de frecuencia equiespaciadas. Cada banda tiene una subportadora que transporta una porción de la información.

Cada una de estas subportadoras es ortogonal al resto, con lo que se consigue que, aunque estén traslapadas, como se observa en la Fig. 5, no exista inferencia entre ellas.

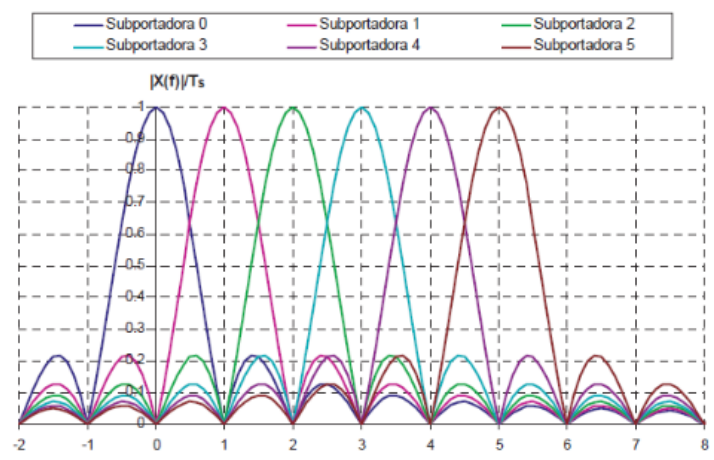

FIG. 5. Ejemplo del espectro correspondiente a seis subportadoras OFDM.

El flujo de datos se divide en $N$ (el número de subportadoras asignadas) flujos paralelos que transmiten a una tasa $\mathrm{N}$ veces menor. De esta manera, se consigue transmitir a la misma velocidad, pero con flujos de información más lentos, lo que hace más improbable que se produzcan errores de transmisión. Cuando mayor es el número de 
subportadoras del que dispone un usuario, mayor es la velocidad o la calidad de la transmisión.

El hecho de poder asignar fácilmente subportadoras a los usuarios, permite repartir el ancho de banda de manera óptima, consiguiendo una buena relación velocidad/calidad de transmisión global. Gracias al uso de OFDM en LTE se pueden alcanzar una tasa de transmisión de $300 \mathrm{Mbps}$ en bajada y 75 Mbps en subida, pero no se llegó al límite de 1 Gbps que exige el estándar para cuarta generación.

Por otro lado se debe hablar acerca de la técnica SCFDMA que LTE utiliza para acceso múltiple en el canal ascendente. Se trata de una técnica muy similar a OFDMA, pero se utiliza en el canal de subida porque permite realizar un uso más eficiente de la energía, reduciendo el consumo de potencia del terminal y consecuentemente incrementando la duración de la batería, algo que también se podría conseguir con OFDMA, mediante la aplicación de determinadas técnicas, pero a costa de un incremento en la complejidad computacional. Por este motivo, en el sistema LTE la técnica de acceso múltiple OFDMA se emplea solo en enlace descendente, ya que en la estación base no son tan críticos ni la eficiencia ni el costo de los amplificadores, no así del lado del enlace ascendente donde se ha optado por utilizar la técnica de acceso con portadora única SC-FDMA, por los motivos que ya fueron expuestos con anterioridad.

Finalmente en este análisis de tecnologías de nivel físico que soportan los sistemas LTE se debe mencionar las estructuras asociadas al uso de varias antenas, donde la diversidad en espacio es la primera estructura ampliamente usada en las comunicaciones móviles. La idea básica es que con el uso de diversas antenas receptoras MR se puede lograr una mejor recepción, logrando una señal combinada con un mayor nivel de potencia y de este modo recuperar la señal de información modulada en mejores condiciones con respecto al ruido. A esta estructura de antenas se le conoce como SIMO (Single Input Multiple Output).

La diversidad en espacio puede verse también desde el lado del transmisor, de modo que la señal es transmitida por un conjunto de antenas MT. Esta estrategia conduce a un esquema MISO (Multiple Input Single Output).

Una nueva estrategia derivada de explotar la presencia de múltiples MT, antenas de transmisión, y MR, antenas de recepción, surge con el concepto de MIMO (Multiple Input Multiple Output), cuya diferencia conceptual con respecto a las estrategias anteriores radica en el hecho de que con MIMO se pretende explotar la variación espacial del canal móvil creando $\mathrm{N}$ caminos de transmisión paralelos y desacoplados entre el emisor y el receptor (pudiendo enviar información diferente por cada camino), lo cual se conoce con el nombre de multiplexado espacial, y es la implementación utilizada por los sistemas LTE.

La principal ventaja de utilizar MIMO es que aumenta el rendimiento y la fiabilidad sin necesidad de incrementar el ancho de banda o la potencia de transmisión.

\section{F. LTE Advanced}

El estándar LTE-Advanced es la sucesión cronológica que supone las mejoras técnicas para la especificación LTE.

LTE-Advanced está especificado en el Release-10 del 3GPP (véase tabla I), y responde a la evolución de un conjunto de especificaciones que van más allá de IMT2000, conocido como IMT-Advanced y referido como 4G.

Las especificaciones para LTE-Advanced entre otras se pueden resumir de la siguiente manera [7]:

- Soporte de velocidades pico de hasta 1 Gbps en downlink y 500 Mbps en uplink.

- Agregación de banda hasta $100 \mathrm{MHz}$, por ejemplo a partir de agregar múltiples componentes de $20 \mathrm{MHz}$ para poder alcanzar un ancho de banda de $100 \mathrm{MHz}$ y así proporcionar las velocidades de transmisión más elevadas previstas en los requerimientos.

- Extensión de soluciones multi-antena, con hasta 8 niveles en el downlink y 4 niveles en el uplink, para así incrementar las velocidades de transmisión alcanzables sobre el enlace.

- Coordinated multipoint transmission and reception (CoMP), que permite mejorar las prestaciones observables en el extremo de la célula a través de efectuar la transmisión/recepción desde distintas células. CoMP es un término relativamente general, que incluye diferentes tipos de coordinación (packet scheduling, beamforming, etc.) entre transceptores separados geográficamente.

- Repetidores, como mecanismo para mejorar la cobertura y reducir el coste de despliegue.

El análisis expuesto denota una tendencia progresiva hacia una mejora constante en términos de tecnología que se traducen en más y mejores servicios de comuicación. No queda sino estar pendientes a las próximas implementaciones que se den en giro a esta convergencia generacional de móviles, ya que implican la implementación de servicios hasta ahora no disponibles como video y tv en alta definición, juegos en línea y toda la gama de servicios que puede ofrecer la multimedia, además de servicios propios de las redes de conmutación de paquetes como Voz sobre IP; quedamos a la expectativa del advenimiento de la quinta generación $(5 \mathrm{G})$, que de seguro, vendrá llena de sorpresas y mejores experiencias para sus usuarios.

\section{CONCLUSIONES}

Las comunicaciones móviles han marcado un hito en la historia de la humanidad proveyendo en un principio la comodidad que conlleva el poder comunicarse con otras personas a través de dispositivos inalámbricos de gran cobertura.

La naturaleza humana nos obliga a superar nuestros propios logros, y lo que para una determinada época representa un maravilloso descubrimiento, en poco tiempo se convierte en algo obsoleto que pocos quieren usar.

Eso es precisamente lo que ha ocurrido con las comunicaciones móviles y ese afán de cambio y de superación 
del hombre ha permitido que un período de tiempo relativamente corto (poco más de dos décadas) hayamos sido testigos de esta transformación tecnológica en torno a un campo de la ciencia que no para de crecer cada día.

Los servicios que se ofrecen al usuario a través de las redes móviles son cada vez más diversos, pero éstos demandan mayores recursos para que puedan ser soportados.

El consumismo que caracteriza las actuales sociedades es el motor que impulsa estos cambios tecnológicos tan acelerados en respuesta a mercados cada vez más exigentes en términos de velocidad, contenidos y modelos tecnológicos de vanguardia.

Existe un exagerado nivel de obsolescencia tecnológica que un principio podrían parecer signos de avance y superación, pero quizá por detrás conlleva un serio problema social, donde las masas nos vemos cautivadas por agresivas campañas de mercadeo por parte de las grandes compañías, que nos obliga a siempre querer contar con el último modelo, el más rápido, con acceso ininterrumpido; pero ¿hasta qué punto, esto ha sido realmente necesario?

\section{REFERENCIAS}

[1] E. Martínez, "La evolución de la telefonía móvil," articulo publicado en la revista RED, 2001.

[2] B. A. Series, "Mobile network evolution: a revolution on the move," IEEE Communications magazine, p. 104, 2002.

[3] 3GPP, “GPRS \& EDGE." http://www.3gpp.org/ technologies/keywords-acronyms/102-gprs-edge, 2016. [Online; accessed 06-Abril-2016].

[4] R. Rodriguez, M. O. Rodríguez Gámez, M. R. Hernández Perdomo, L. L. Torno Hidalgo, L. L. García Escalona, et al., "Telefonía móvil celular: origen, evolución, perspectivas.," Ciencias Holguín, vol. 11, no. 1, 2010.

[5] 3GPP, “3GPP \& RELEASES.” http://www.3gpp.org/ specifications/67-releases, 2016. [Online; accessed 26Mayo-2016].

[6] C. Serra, "Arquitectura general del sistema lte," articulo publicado en la revista Telem@tica, 2013.

[7] R. Agusti Comes, F. Bernardo Alvarez, F.-j. Casadevall Palacio, R. A. Ferrus Ferre, J. Perez Romero, and J. O. Sallent Roig, "Lte: Nuevas tendencias en comunicaciones móviles," 2010.

Recibido: 16 de enero de 2017

Aceptado: 09 de marzo de 2017

Jhovany Santacruz: Magíster en Redes de Comunicaciones. Catedrático y Subdirector de la carrera de Ingeniería de Sistemas de la Universidad Católica de Cuenca, Extensión Cañar. Consultor informático en proyectos de desarrollo de software.

Rafael García: Magíster en Educación. Catedrático Universitario. Subdirector de Investigación y Publicaciones de la Universidad Católica de Cuenca. Correo electrónico: regarciaa@ucacue.edu.ec 
